Цитирование: Бычков М.Б., Абдуллаев А.Г., Багрова С.Г., Барболина Т.Д., Борисова Т.Н., Владимирова Л.Ю. и соавт. Практические рекомендации по лекарственному лечению мезотелиомы плевры, брюшины и других локализаций. Злокачественные опухоли : Практические рекомендации RUSSCO \#3s2, 2021 (том 11). 04

\title{
П ПРАКТИЧЕСКИЕ РЕКОМЕНДАЦИИ ПО ЛЕКАРСТВЕННОМУ ЛЕЧЕНИЮ МЕЗОТЕЛИОМЫ ПЛЕВРЫ, БРЮШИНЫ И ДРУГИХ ЛОКАЛИЗАЦИЙ
}

Коллектив авторов: Бычков М.Б., Абдуллаев А.Г., Багрова С.Г., Барболина Т.Д., Борисова Т.Н., Владимирова Л.Ю., Деньгина Н.В., Левченко Е.В., Сакаева Д.Д.

Ключевые слова: мезотелиома, канцерогенные факторы, факторы прогноза, химиотерапия, комбинированное лечение, опухолевые плевриты, тримодальная терапия, асцит

Мезотелиома - злокачественная агрессивно растущая опухоль, которая возникает при трансформации мезотелиальных клеток, выстилающих естественные полости организма, и характеризуется поражением плевры, брюшины, перикарда и оболочек яичка. Заболеваемость мезотелиомой плевры (МП) значительно варьирует от 8 случаев на 100000 населения в Англии до 3,1 - в Австралии, 1 - в США, Испании, Польше. Частота МП увеличивается в последние годы в России, странах Западной Европы, Китае, Индии. Пик заболеваемости ожидается в 2020-2025 гг. Мужчины болеют чаще в соотношении 6:1. В РФ вопросу эпидемиологии мезотелиом не уделяется должного внимания. Статистических данных по заболеваемости и смертности нет.

В этиологии МП основное значение (80\% случаев) имеет контакт с асбестом, применяемым в строительной практике. Проследить связь между развитием болезни и контактом с асбестом подчас очень трудно, так как латентный период до клинической манифестации заболевания может достигать 40 лет. Кроме асбеста в развитии мезотелиомы плевры также имеют значение другие химические канцерогены (силикаты, бериллий, жидкий парафин), ионизирующее излучение (описаны случаи развития мезотелиомы через 20-30 лет после лучевой терапии лимфогранулематоза). Имеет значение генетическая предрасположенность (мутации BRCA1-ассоциированного белка BAP-1, позитивный PD-L1), вирус SV-40, гены которого экспрессируются у $60 \%$ больных МП. Для мезотелиом других локализаций наличие таких причинно-следственных связей не доказано.

\section{1. ОПРЕДЕЛЕНИЕ СТАДИИ}

Для стадирования заболевания используется классификация, предложенная Международной группой по изучению мезотелиомы (IMIG), (АJCC, 8 издание, 2017 г.). Стадиро- 
вание по TNM применимо только к мезотелиоме плевры (табл. 1 и 2). Несмотря на то, что выпот в плевральной полости при МП встречается у 40-70\% больных, при стадировании наличие плеврита не учитывается.

Таблица 1. Определение категорий TNM при мезотелиоме плевры

\begin{tabular}{|c|c|}
\hline \multicolumn{2}{|c|}{ Первичная опухоль } \\
\hline $\mathrm{Tx}$ & Первичная опухоль не может быть оценена \\
\hline TO & Первичная опухоль не определяется \\
\hline T1 & $\begin{array}{l}\text { Опухоль ограничена париетальной плеврой на стороне поражения в сочетании или без вовлечения } \\
\text { висцеральной, медиастинальной, диафрагмальной плевры }\end{array}$ \\
\hline $\mathrm{T} 2$ & $\begin{array}{l}\text { Опухоль прорастает любую поверхность париетальной плевры на стороне поражения. Присутствует } \\
\text { одна из следующих характеристик: } \\
\text { • } \quad \text { инвазия в диафрагмальные мышцы } \\
\text { • } \quad \text { инвазия в подлежащую паренхиму легкого }\end{array}$ \\
\hline T3 & $\begin{array}{l}\text { Опухоль локально распространенная, но потенциально резектабельная, прорастает любую поверхность } \\
\text { париетальной плевры на стороне поражения. Присутствует одна из следующих характеристик: } \\
\text { • } \quad \text { инвазия во внутригрудную фасцию } \\
\text { • } \quad \text { инвазия в жировую клетчатку средостения } \\
\text { • } \quad \text { единичный опухолевый узел, прорастающий в мягкие ткани грудной клетки } \\
\text { • } \quad \text { не трансмуральное поражение перикарда }\end{array}$ \\
\hline T4 & $\begin{array}{l}\text { Местно-распространенный, но технически не резектабельный процесс. Опухоль прорастает все } \\
\text { поверхности париетальной плевры на стороне поражения. Присутствует одна из следующих } \\
\text { характеристик: }\end{array}$ \\
\hline & 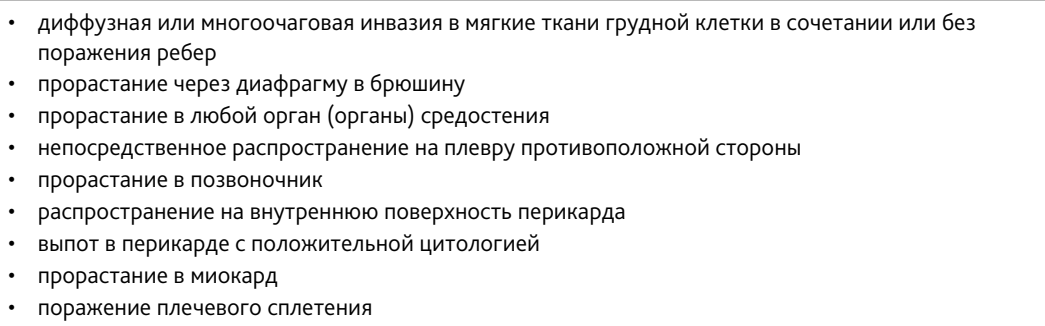 \\
\hline \multicolumn{2}{|c|}{ Регионарные лимфатические узлы1, 2} \\
\hline $\mathrm{Nx}$ & Регионарные лимфатические узлы не могут быть оценены \\
\hline No & Нет метастазов в регионарных лимфатических узлах \\
\hline N1 & $\begin{array}{l}\text { Метастазы в бронхопульмональных лимфатических узлах (узле) и/или лимфатических узлах (узле) } \\
\text { корня легкого на стороне поражения, медиастинальных лимфатических узлов, включая внутренние } \\
\text { маммарные, перидиафрагмальные, перикардиальной клетчатки и интеркостальные на стороне } \\
\text { поражения }\end{array}$ \\
\hline N2 & $\begin{array}{l}\text { Метастазы в надключичных лимфатических узлах (узле) на стороне поражения или противополож- } \\
\text { ной стороне, контралатеральных медиастинальных лимфатических узлах }\end{array}$ \\
\hline
\end{tabular}




\begin{tabular}{|l|l|}
\hline \multicolumn{2}{|l|}{ Отдаленные метастазы } \\
\hline M0 & Нет отдаленных метастазов \\
\hline M1 & Есть отдаленные метастазы (печень, паренхима легких, кости, головной мозг) \\
\hline
\end{tabular}

1 Для мезотелиомы брюшины регионарными являются забрюшинные лимфатические узлы.

${ }^{2}$ Длямезотелиомы оболочек яичка регионарными являются паховые и подвздошные лимфатические узлы.

Таблица 2. Группировка мезотелиомы плевры по стадиям

\begin{tabular}{|l|l|l|l|}
\hline Стадия & T & N & M \\
\hline Ia & T1 & N0 & M0 \\
\hline Ib & T2-3 & N0 & M0 \\
\hline II & T1-2 & N1 & M0 \\
\hline IIIA & T3 & N1 & M0 \\
\hline IIIB & T1-3 & N2 & M0 \\
\hline & T4 & Nлюбая & M0 \\
\hline IV & Тлюбая & Nлюбая & M1 \\
\hline
\end{tabular}

\section{2. ДИАГНОСТИКА}

Скрининговых программ для выявления мезотелиомы нет. Диагностика МП сложна в плане дифференциального диагноза с метастазами по плевре других злокачественных опухолей (рака молочной железы, легкого, почки, толстой кишки, яичников) и поражением плевры синовиальной саркомой. Мезотелиому брюшины дифференцируют с канцероматозом брюшины при раке яичников, раке желудка; мезотелиому оболочек яичка с доброкачественным гидроцеле или герминогенными опухолями. Мезотелиома перикарда схожа по клинической картине с хронической сердечной недостаточностью.

Торакоскопия или лапароскопия обязательны в диагностическом алгоритме, т. к. помогают визуально оценить степень диссеминации, операбельность и выполнить биопсию с ИГХ исследованием для точного определения гистологического типа опухоли. Мезотелиальные клетки отличаются от фибробластических и эпителиальных только при электронной микроскопии и иммунофенотипировании. ИГХ-панель выполняется в позитивном и в негативном окрашивании. Характерно наличие кальретинина, виментина, мезотелина и антигена WT-1. Не рекомендуется устанавливать диагноз только на основании цитологического исследования, так как при этом методе точный диагноз устанавливается только в $26 \%$ случаев.

Первичное обследование должно проводиться до начала лечения для определения распространенности болезни, стадирования и определения тактики лечения. План обследования больных мезотелиомой: 
- сбор анамнеза и физикальный осмотр;

- общий анализ крови (гемоглобин, общее число лейкоцитов, лейкоцитарная формула, тромбоциты);

- биохимический анализ крови (креатинин, мочевина, АЛТ, АСТ) и коагулограмма;

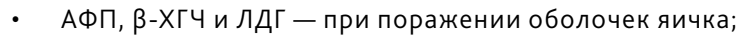

- КТ органов грудной клетки и брюшной полости с в/в контрастированием;

- УзИ шейно-надключичных, подключичных лимфатических узлов, паховых лимфатических узлов - при мезотелиоме оболочек яичка, органов брюшной полости, забрюшинного пространства и малого таза;

- рентгенологическое исследование органов грудной клетки (лишь для определения уровня гидроторакса); диагноз МП не может быть установлен по обычной рентгенограмме;

- радиоизотопное исследование скелета (при подозрении на поражение костей);

- КТ или МРТ головного мозга с контрастным усилением (при наличии неврологических симптомов или при ранней стадии для исключения поражения).

МРТ является методом уточняющей диагностики при оценке местного распространения опухоли. КТ и МРТ играют важную роль в оценке распространенности опухоли по диафрагме, средостению, верхней части грудной клетки. При неинвазивной стадии очень трудно провести различия между Т1 и Т2.

- УзДг сосудов шеи и нижних конечностей (при гиперкоагуляции);

- ЭКГи Эхо-КГ при поражении перикарда;

- торакоскопическая биопсия плевры или перикарда/лапароскопическая биопсия брюшины, биопсия паховых лимфатических узлов при мезотелиоме оболочек яичка;

- ИГХи/или ИЦХ исследование;

- выполнение ПЭТ-КТ с ФДГ с целью первичного стадирования не рекомендуется, но является дополнительным методом в диагностике поражения плевры, может использоваться в качестве контроля эффективности лечения, перед планированием ЛТ.

Гистологические подтипы мезотелиомы включают в себя 3 формы: эпителиоидную, бифазную и саркоматоидную. Наиболее часто встречается эпителиоидная (50-70\% случаев), реже - бифазная (20-25\% случаев) и саркоматоидная (7-20\% случаев) формы. у больных с эпителиоидной формой отмечаются лучшие отдаленные результаты лечения, чем у пациентов с бифазной и саркоматоидной формами.

\section{3. ПРОГНОСТИЧЕСКИЕ ФАКТОРЫ}

К числу прогностических факторов при МП относят возраст, пол, стадию заболевания, гистологический тип опухоли, ответ на лечение, общесоматический статус по шкале ECOG, потерю веса, исходный уровень гемоглобина и лейкоцитов (табл. 3). Экспрессия PD-L1 - прогностический фактор для МП. Отсутствие экспрессии PD-L1 ассоциируется с увеличением выживаемости). 
Таблица 3. Прогностические факторы при мезотелиоме плевры

\begin{tabular}{|l|l|l|}
\hline Параметр & $\begin{array}{l}\text { Благоприятный } \\
\text { прогностический фактор }\end{array}$ & $\begin{array}{l}\text { Неблагоприятный } \\
\text { прогностический фактор }\end{array}$ \\
\hline Возраст & $<75$ лет & $\geq 75$ лет \\
\hline Боль в грудной клетке & Нет & Есть \\
\hline Количество тромбоцитов & $<400 \times 109 / л$ & $\geq 400 \times 109 / л$ \\
\hline Гемоглобин $\geq 14,6$ г/дл & $<11,2$ г/дл & \\
\hline Потеря массы тела & Нет & Есть \\
\hline ЕСОС & 0 & $1-2$ \\
\hline Гистологический вариант & Эпителиоидный & Неэпителиоидный \\
\hline Пол & Женский & Мужской \\
\hline Лейкоциты & $<8,3 \times 109 / л$ & $\geq 8,3 \times 109 / л$ \\
\hline Точность диагностики & Максимальная & Минимальная \\
\hline РD-L1 & Отрицательный<5\% & Положительный $\geq 5 \%$ \\
\hline
\end{tabular}

\section{4. ЛЕЧЕНИЕ}

\subsection{I-IIIA стадии}

\subsection{1. Хирургическое лечение}

Хирургический метод лечения предпочтителен для ранних стадий. Объем операции:

- экстраплевральная пневмонэктомия (ЭПП)

- плеврэктомия или декортикация (ПЭ, ПД)

- плевродез как метод лечения рецидивирующего плеврита.

Операция проводится в многопрофильном лечебном учреждении, опытным хирургом. Требования, предъявляемые к пациентам до операции:

- удовлетворительное общее состояние

- сохранная легочно-сердечная функция

- эпителиоидный тип опухоли

- $\mathrm{NO}-1$

Целесообразность хирургического вмешательства при саркоматоидном гистологическом варианте опухоли и поражении лимфатических узлов N2 спорна.

Плеврэктомия или декортикация в сравнении с ЭПП сопровождается лучшими показателями послеоперационной летальности (0-4\% против 5-7\%, соответственно) [Flores R.M., 2008, Weder W. et al, 2011]. Метаанализ, в который включено 2903 оперированных пациентов с МП, подтвердил лучшие непосредственные результаты ПД в сравнении с ЭПП, однако отсутствие статистически значимых различий в 2-х летней выживаемости 
авторы объяснили геторогенностью групп [Taioli E, 2015]. Следует иметь ввиду, что не всем пациентам с МП возможно выполнение ПД из-за наличия массивной инвазии опухоли в паренхиму легкого или буллезной эмфиземы, при которых когда удаление висцеральной плевры сопряжено с массивным воздухоистечением.

Есть данные об эффективности адъювантных интраоперационных методов лечения, включая гипертермическую интраоперационную ХТ (ГИОХ или HIOC) [Baldini EH, et al. 1997], экспозицию в течение 15 мин. подогретого до 40-41ㄷ раствора бетадина (PVP-I) [Lang-Lazdunski L, et al. 2012] и ФДТ [Castano AP, et al. 2006], нацеленных на микрометастазы и направленных на улучшение местного контроля. Решение о таких дополнительных методах воздействия принимается на междисциплинарном консилиуме.

Париетальная плеврэктомия или плевродез показаны для купирования рецидивирующего плеврита. ПЭ не увеличивает выживаемость по сравнению с ЭПП, но сокращает частоту рецидивов накопления плевральной жидкости лучше, чем плевродез тальком. При перикардите со значительным объемом жидкости (при ЭхоКГ в фазе диастолы расхождение листков перикарда >20 мм) рекомендуется перикардиоцентез.

При мезотелиоме брюшины рекомендуется комбинированный подход: циторедуктивная операция +интраперитонеальная гипертермическая химиотерапия HIPEC (Hyperthermic intraperitoneal chemotherapy), при которых 5-летняя общая выживаемость достигается у $52 \%$ больных. При невозможности выполнения НІРЕС проводится отсроченная нормотермическая внутрибрюшинная ХТ.

При мезотелиоме оболочек яичка оптимальным объемом операции считается орхфуникулэктомия (ОФЭ) в сочетании или без паховой лимфодиссекции. Локальная резекция стенки оболочки яичка связана с локальной частотой рецидивов $36 \%$, иля местного контроля часто требуется гемискротэктомия, тогда как местный рецидив после орхидэктомии отмечается у 10,5-11,5\% пациентов. Поскольку в большинстве случаев злокачественная мезотелиома диагностируется интраоперационно или в результате патоморфологического исследования удаленных образцов, то в случае первоначально выполненной гемискротэктомии на втором этапе объем оперативного вмешательства расширяют до радикального. Вопрос о необходимости пахово-подвздошной лимфодиссекции остается дискутабельным

Адъювантная химиотерапия показана:

- после ЭПП и радикальной ПЭ при мезотелиоме плевры;

- после циторедуктивной перитонэктомии при мезотелиоме брюшины.

После ОФЭ при мезотелиоме оболочек яичка и после перикардэктомии при мезотелиоме перикарда вопрос об адъювантной ХТ решается индивидуально.

\subsection{2. Комбинированное лечение}

Хирургическое лечение может рассматриваться при МП как компонент тримодальной терапии. Если операция RO сомнительна, настоятельно рекомендуется проводить комбинированную терапию с XТ и/или лТ. Показания к трехкомпонентному лечению определяются индивидуально, исходя из общего состояния пациента, распространенности процесса, ожидаемой эффективности ХT, технических и кадровых возможностей стацио- 
нара. С учетом данных рандомизированного исследования MARS (2011 г.) оптимальным объемом хирургического этапа при комбинированном лечении считается ПД. Алгоритм диагностики и лечения ранних стадий МП представлен на рис. 1.

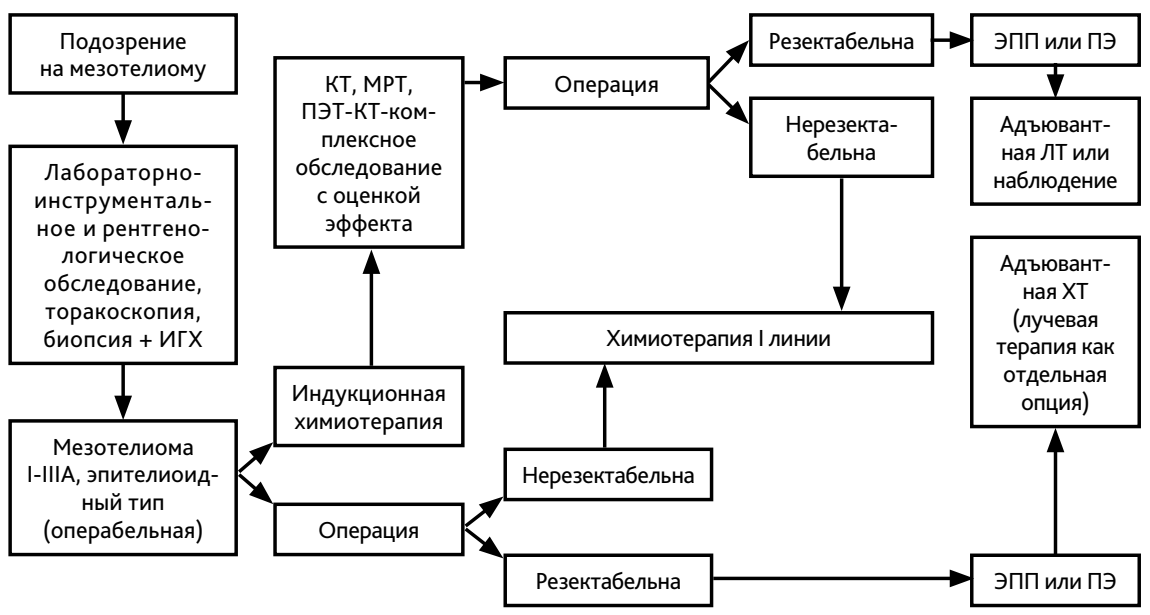

Рисунок 1. Алгоритм диагностики и лечения локализованной мезотелиомы плевры.

\subsection{IIIB-IV стадия}

\subsection{1. Химиотерапия}

При невозможном выполнении оперативного вмешательства при ранней стадии, бифазном или саркоматоидном подтипе, а также при распространенном опухолевом процессе применяется XТ. В последние годы выделяют два основных режима XТ первой линии - это платиносодержащие режимы в комбинации с пеметрекседом или гемцитабином. Стандартов второй и последующих линий лечения нет. Основные и альтернативные режимы лекарственного лечения представлены в табл. 4.

Таблица 4. Режимы химиотерапии, рекомендуемые при мезотелиоме

\begin{tabular}{|c|c|}
\hline $\begin{array}{l}\text { Схема } \\
\text { лечения }\end{array}$ & Режим химиотерапии \\
\hline PP1 & Пеметрексед 500 мг/м² в 1-й день + цисплатин 75 мг/м² в 1-й день, каждые 21 день \\
\hline PC1 & Пеметрексед 500 мг/м² в 1-й день + карбоплатин AUC-5 (6) в 1-й день, каждые 21 день \\
\hline GP & Гемцитабин 1000-1250 мг/м² в 1-й и 8-й дни + цисплатин 75 мг/м² в 1-й день, каждые 21 день \\
\hline GC & Гемцитабин 1000-1250 мг/м² в 1-й и 8-й дни + карбоплатин AUC-5 (6) в 1-й день, каждые 21 день \\
\hline
\end{tabular}




\begin{tabular}{|c|c|}
\hline GemOx & $\begin{array}{l}\text { Гемцитабин } 1000 \text { мг/м² в 1-й и 8-й дни + оксалиплатин } 80 \text { мг/м² в 1-й и 8-й дни, } \\
\text { каждые } 21 \text { день }\end{array}$ \\
\hline IP & Иринотекан 65 мг/м² в 1-й и 8-й дни + цисплатин 75 мг/м² в 1-й день, каждые 21 день \\
\hline $\mathrm{CP}$ & Паклитаксел 175 мг/м² в 1-й день + карбоплатин AUC-5 (6) в 1-й день, каждые 21 день \\
\hline AP & Доксорубицин 60 мг/м² в 1-й день + цисплатин 60 мг/м² в 1-й день, каждые 21 день \\
\hline Винорелбин & 20 мг/м² в 1-й, 8-й, 15-й дни, каждые 28 дней \\
\hline Винорелбин & 25-30 мг/м² в 1-й и 8-й дни, каждые 21 день \\
\hline
\end{tabular}

1 оптимальный объемлечения

Рекомендуется проведение 6 курсов XТ с последующим динамическим наблюдением. Смена режима ХТ должна проводиться только при доказанном прогрессировании заболевания или в случае непереносимости лекарственного лечения. В случае прогрессирования болезни в течение $\geq 6$ мес. от последнего курса ХТ желательна реиндукция режима первой линии лечения. При раннем прогрессировании на фоне лечения или в ближайшие 6 мес. после завершения XT рекомендуется переход на вторую линию. Алгоритм диагностики и лечения диссеминированной мезотелиомы плевры представлен на рис. 2.

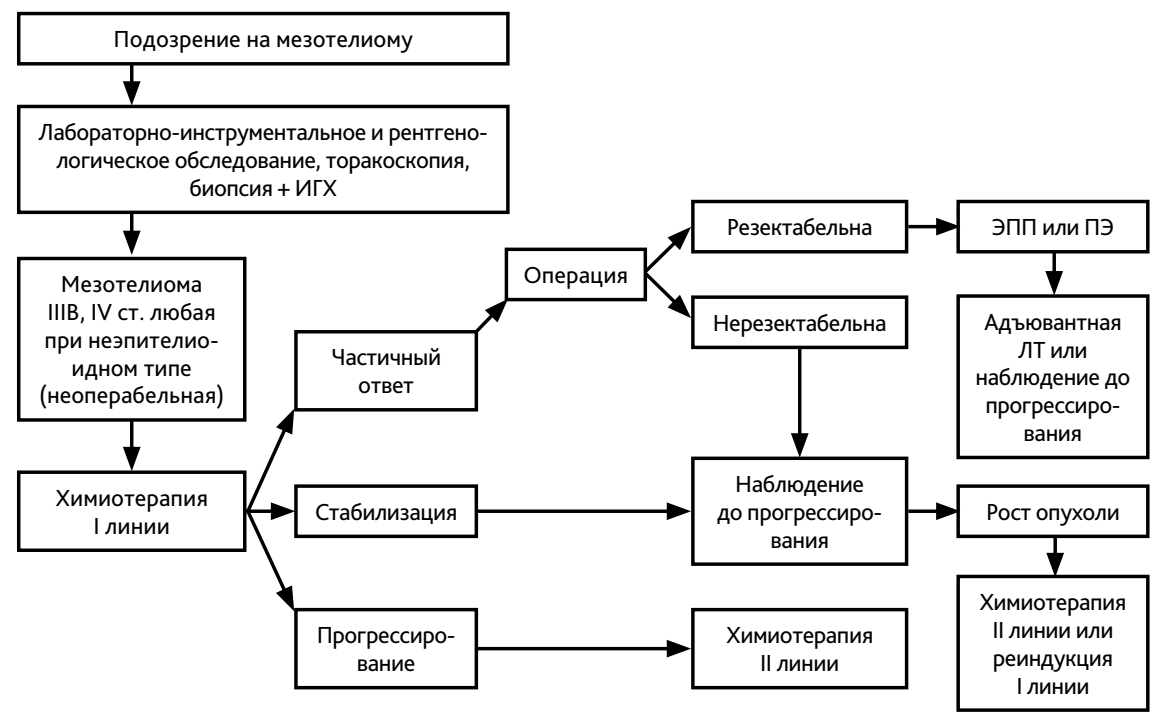

Рисунок 2. Алгоритм диагностики и лечения диссеминированной мезотелиомы плевры. 


\subsection{2. Плевроцентез или лапароцентез и плевродез как методы лечения рецидивирующего гидроторакса и накопления асцита}

\subsubsection{1. Показания к выполнению}

Для МП характерно развитие гидроторакса, который ухудшает качество жизни пациента и создает угрозу жизни. Эвакуация жидкости из плевральной полости проводится с помощью пункции - плевроцентеза, который представляет собой исключительно симптоматический метод лечения.

Показаниями для плевроцентеза являются:

- наличие жидкости в плевральной полости с уровнем выше 2-3 ребра при счете спереди; - симптомы легочно-сердечной недостаточности: выраженная одышка в покое, приглушенный ритм сердца, распирающая боль в грудной клетке и т. д.

При отсутствии клинических симптомов выполнение плевроцентеза не показано.

У $70 \%$ больных отмечается повторное накопление жидкости. Показанием к проведению плевродеза является постоянное накопление жидкости, требующее повторных плевральных пункций с частотой 3 раза в месяц. Применение плевросклерозирующих средств приводит к облитерации плевральной полости за счет асептического воспаления плевральных листков, их склеивания и последующего сращения париетальной и висцеральной плевры с прекращением продукции плеврального выпота.

Показанием к лапароцентезу является значительное скопление асцитической жидкости в брюшной полости с формированием так называемого напряженного асцита.

\subsubsection{2. Методика выполнения}

При плевроцентезе под местной анестезией в положении больного сидя с опорой вперед на спинку стула для максимального расширения межреберных промежутков выполняют пункцию плевральной полости и ее дренирование (с помощью специального набора, например, плеврокана) и максимально эвакуируют жидкость (пассивно или активно с использованием шприца). Желательна разметка места прокола с помощью УзИ. Прокол проводится, как правило, в проекции 7-8 ребер по лопаточной линии. Появление болевых ощущений и/или кашля косвенно указывают на расправление легкого и соприкосновение листков плевры. При большом объеме жидкости целесообразно каждые 1,5-2 часа временно перекрывать дренаж во избежание быстрого смещения органов средостения в противоположную сторону. После окончания процедуры выполняют рентгенологическое исследование с целью контроля расправления легкого. При недостаточном расправлении легкого и формировании остаточной полости (вследствие длительного коллапса легкого жидкостью и его фиксации к грудной стенке или к диафрагме) введение склерозирующих препаратов бесперспективно. При эффективном осушении плевральной полости, когда жидкость остается в незначительном количестве только в плевральном синусе, легкое расправлено, признаков пневмоторакса нет, можно вводить склерозирующие препараты. Поскольку введение препаратов может сопровождаться тошнотой, рвотой и болевыми ощущениями вплоть до очень сильных, введение их должно предваряться в/в введением 
антиэметиков в стандартных дозах и внутриплевральной анестезией (50-100 мл 0,5\% раствора новокаина или 0,25\% лидокаина). При внутриплевральном введении цисплатина необходима также гипергидратация (1,5-2 л физиологического раствора в/в). После выполнения этих процедур плеврокан (дренаж) перекрывают на 1-2 часа. По истечении указанного времени дренаж следует открыть для свободного истечения остаточной жидкости. Затем необходимо ввести лекарственный агент через дренажную трубку и удалить дренаж. Неудачи данной методики связаны в основном с недостаточно тщательным дренированием плевральной полости.

Наиболее эффективным склерозирующим агентом признан тальк. Введение его через плевральный катетер в виде суспензии (4-5 г стерильного талька +20 мл 0,5\% раствора лидокаина) эффективно у $90 \%$ больных. Возможна также инсуфляция порошка талька при торакоскопии. Препараты, рекомендуемые для проведения плевродеза, представлены в табл. 5.

Таблица 5. Препараты, рекомендуемые для проведения плевродеза

\begin{tabular}{|l|l|}
\hline Препараты & Доза, рекомендуемая на одно введение1 \\
\hline Тальк & $2-5$ г \\
\hline Блеомицин & $15-30$ мг \\
\hline Цисплатин & 50 мг \\
\hline
\end{tabular}

1 Выбор дозы зависит от массы тела пациента. Введение лекарственных средств внутриплеврально рекомендуется осуществлять не чаще 1 раза в неделю, не более 3 введений в общей сложности.

Осложнения внутриплеврального введения лекарственных средств:

- болевой синдром;

- усиление одышки;

- гипертермия;

- ателектаз легкого;

- миелосупрессия;

- пневмония;

- эмпиема плевры;

- легочная и сердечно-легочная недостаточность.

Плевродез является малым хирургическим вмешательством и должен проводиться в только в стационаре, хирургом с участием химиотерапевта. Следует учитывать системное воздействие цитотоксических веществ, вводимых внутриплеврально, и по возможности не выполнять эту процедуру одновременно с ХТ во избежание усиления токсичности.

Лапароцентез также проводят в положении больного сидя, пациент должен отклониться назад и облокотиться на руки; на колени больного заранее кладут клеенку (пеленку) в виде фартука. Соблюдение асептики обязательно. Предварительно освобождают кишечник и мочевой пузырь. Под местной инфильтрационной анестезией 0,5\% раствором новокаина (лидокаина) на 3 пальца ниже пупка и на 2 см левее белой линии живота 
выполняют пункцию при помощи плеврокана. Жидкость выпускают без форсирования, ориентируясь на общее состояние больного. При мезотелиоме брюшины характерно, особенно при многократных лапароцентезах, формирование желеобразного состояния асцита, что затрудняет его эвакуацию. В этом случае приходится активно эвакуировать жидкость шприцем. По завершении эвакуации асцитической жидкости извлекают трубку плеврокана и на рану брюшной стенки накладывают давящую асептическую повязку. При подтекании асцита возможно наложение одного шва и марлевой повязки. Живот целесообразно «утянуть» с некоторым натяжением, чтобы сохранить привычное для больного внутрибрюшное давление.

Лапароцентез противопоказан при:

- нарушениях свертываемости крови ввиду риска кровотечения;

- тяжелой спаечной болезни брюшной полости;

- метеоризме;

- вентральной грыже после предыдущих оперативных вмешательств;

- риске травмы кишечника, крупной опухоли.

Не рекомендуется проводить лапароцентез близко к области мочевого пузыря, пальпируемого опухолевого образования. Наличие спаек-относительное противопоказание, но сама по себе спаечная болезнь предполагает высокий риск повреждения сосудов и органов брюшной полости, поэтому показания к лапароцентезу в этом случае оцениваются индивидуально.

\subsection{3. Лучевая терапия}

ЛТ как самостоятельный метод лечения не используется. При проведении адъювантной лТ поле облучения обязательно включает париетальную плевру при декортикации легкого, границы хирургических клипс после ЭПП и участки с возможными остаточными проявлениями. ЛТ лимфатических узлов средостения и надключичных зон не рекомендуется. Послеоперационная ЛТ возможна у пациентов в удовлетворительном состоянии (ECOG 0-1), с сохранной легочной функцией, при которой пациент не нуждается в кислородотерапии, без признаков почечной недостаточности, при отсутствии отдаленных метастазов. ЛТ проводится квалифицированными специалистами в специализированных центрах с применением методик IMRT, VMAT, TomoTherapy, которые исключают бо́льшую часть легкого из облучения. Рекомендуемые дозы лт указаны в табл. 6.

Таблица 6. Рекомендуемые дозы лучевой терапии при мезотелиоме плевры

\begin{tabular}{|c|c|c|c|}
\hline Назначение & сод & РД & $\begin{array}{l}\text { Длительность } \\
\text { лечения }\end{array}$ \\
\hline \multicolumn{4}{|l|}{ После операции } \\
\hline После ЭПП & $50-60 Г p$ & $1,8-2 \Gamma \mathrm{p}$ & 6-7 нед. \\
\hline После Пэ & $45-54\lceil p$ & $1,8-2 \Gamma \mathrm{p}$ & 5-6 нед. \\
\hline После циторедуктивных (R2) резекций & $50-54 Г p$ & $1,8-2 \Gamma \mathrm{p}$ & 5-6 нед. \\
\hline
\end{tabular}




\begin{tabular}{|l|l|l|l|}
\hline Паллиативная & 20-40 Гр & $3-4$ Гр & $1-2$ нед. \\
\hline С обезболивающей целью на грудную клетку & $30-40$ Гр & $3-4$ Гр & 2 нед. \\
\hline На область асимптомного рецидива & Возможны варианты \\
\hline Метастазы в головном мозге или костях & \multicolumn{2}{|l|}{} \\
\hline
\end{tabular}

При мезотелиоме брюшины ЛТ имеет ограниченные возможности. Данных об эффективности ЛТ при мезотелиоме перикарда или оболочек яичка нет.

\section{5. ТАРГЕТНАЯ И ИММУНОТЕРАПИЯ}

Единственный таргетный препарат, показавший эффективность в лечении мезотелиомыбевацизумаб. Его добавление к ХТ пеметрекседом помогает получить максимально возможную эффективность и увеличить продолжительность жизни. (медиана времени без прогрессирования составила 9,2 против 7,3 мес., медиана продолжительности жизни - 18,8 против 16,1 мес.). Но добавление бевацизумаба к режиму GP не улучшило эффективность лечения у больных МП по данным Kindler et al в многоцентровом рандомизированном двойном слепом плацебо контролируемом исследования фазы II (частота объективных эффектов 24,5\% против 21,8\%, медиана продолжительности жизни 15,6 против 14,7 мес.).

В качестве II-III линии лечения возможна иммунотерапия пембролизумабом при экспрессии PD-L1 $\geq 1 \%$, которая позволяет достичь частоту объективных ответов в $22 \%$ случаев, а контроль роста опухоли - у $76 \%$ пациентов, но не увеличивает время до прогрессирования и общую выживаемость. Медиана продолжительности жизни при использовании пембролизумаба достигает 18 мес. при МП. Пембролизумаб зарегистрирован также в РФ для лечения солидных опухолей при наличии MSI-H, а при мезотелиоме брюшины этот маркер встречается в 1-2\% случаев. Эффективность пембролизумаба при мезотелиоме перикарда и оболочек яичка не изучалась.

В качестве II-III линии лечения возможно также использование иммунотерапии ниволумабом в сочетании или без ипилимумаба, независимо от уровня экспрессии PD-L1. Режимы иммунотерапии представлены в табл. 7.

Таблица 7. Рекомендуемые режимы таргетной и иммунотерапии при мезотелиоме плевры

\begin{tabular}{|l|l|}
\hline Препарат & Режим \\
\hline Бевацизума61 & 15 мг/кг в/в кап. 1 раз в 3 недели \\
\hline Пембролизума6 & 200 мг в/в кап. 1 раз в 3 недели \\
\hline Ниволума6 & 3 мг/кг в/в кап. 1 раз в 14 дней или 240 мг в/в кап. каждые 14 дней не более 2 лет \\
\hline Ниволума6+ ипилимума6 & $\begin{array}{l}\text { Ниволума6 } 3 \text { мг/кг в/в кап. } 1 \text { раз в } 14 \text { дней или } 360 \text { мг в/в кап. каждые } \\
\text { 3 недели + ипилимума6 } 1 \text { мг/кг в/в кап. } 1 \text { раз в } 6 \text { недель не более } 2 \text { лет }\end{array}$ \\
\hline
\end{tabular}

1 в комбинации с ХT 6 курсов, далее - в поддерживающем режиме 1 раз в 3 недели до 1 года или до прогрессирования болезни, что решается индивидуально в каждом конкретном случае. Комбинация «XT+бевацизумаб» не используется в неоадъювантном лечении. 


\section{6. НАБЛЮДЕНИЕ}

После завершения лечения динамическое наблюдение проводится каждые 3 мес. в течение первого года, далее - 1 раз в 6 мес. до продолжительности 5 лет. Обязательными диагностическими процедурами являются КТ грудной клетки или брюшной полости с контрастированием, в зависимости от того, где локализовалась первичная опухоль, УзИ шейно-надключичных, подключичных, паховых лимфатических узлов, органов брюшной полости, забрюшинного пространства и малого таза. 